\title{
Profiling the interactome of protein kinase $C \zeta$ by proteomics and bioinformatics
}

\author{
Chunyu Hou ${ }^{1,2 \dagger}$, Yuan Li ${ }^{1,2 \dagger}$, Huiqin Liu ${ }^{1,2}$, Mengjiao Dang ${ }^{3}$, Guoxuan Qin ${ }^{3}$, Ning Zhang ${ }^{1,2}$ \\ and Ruibing Chen ${ }^{1,2^{*}}$
}

\begin{abstract}
Background: Protein kinase C $\zeta$ (PKC (), an isoform of the atypical protein kinase $C$, is a pivotal regulator in cancer. However, the molecular and cellular mechanisms whereby PKC regulates tumorigenesis and metastasis are still not fully understood. In this study, proteomics and bioinformatics analyses were performed to establish a protein-protein interaction (PPI) network associated with PKCद, laying a stepping stone to further understand the diverse biological roles of PKCद.

Methods: Protein complexes associated with PKC $\zeta$ were purified by co-immunoprecipitation from breast cancer cell MDA-MB-231 and identified by LC-MS/MS. Two biological replicates and two technical replicates were analyzed. The observed proteins were filtered using the CRAPome database to eliminate the potential false positives. The proteomics identification results were combined with PPI database search to construct the interactome network. Gene ontology (GO) and pathway analysis were performed by PANTHER database and DAVID. Next, the interaction between PKCZ and protein phosphatase 2 catalytic subunit alpha (PPP2CA) was validated by co-immunoprecipitation, Western blotting and immunofluorescence. Furthermore, the TCGA database and the COSMIC database were used to analyze the expressions of these two proteins in clinical samples.
\end{abstract}

Results: The PKC centered PPI network containing 178 nodes and 1225 connections was built. Network analysis showed that the identified proteins were significantly associated with several key signaling pathways regulating cancer related cellular processes.

Conclusions: Through combining the proteomics and bioinformatics analyses, a PKCद centered PPI network was constructed, providing a more complete picture regarding the biological roles of PKC $\zeta$ in both cancer regulation and other aspects of cellular biology.

Keywords: Cancer, PKCद; LC-MS/MS, Proteomics, Bioinformatics, Protein-protein interaction

\section{Background}

Protein kinase $\mathrm{C}$ (PKC) is a family of protein serine/ threonine kinases that are involved in the regulation of diverse biological processes, including gene expression, cell differentiation, apoptosis, proliferation, cytoskeleton organization, cell migration, etc. [1-3]. Based on their distinct features, PKCs can be divided into three

\footnotetext{
* Correspondence: chenruibing@tijmu.edu.cn

${ }^{\dagger}$ Equal contributors

${ }^{1}$ Tianjin Medical University Cancer Institute and Hospital, National Clinical Research Center for Cancer, Key Laboratory of Cancer Prevention and

Therapy, Tianjin's Clinical Research Center for Cancer, Tianjin 300070, China

${ }^{2}$ Department of Genetics, School of Basic Medical Sciences, Tianjin Medical

University, Tianjin 300070, China

Full list of author information is available at the end of the article
}

subtypes [4]: the conventional or classic PKCs, such as PKC $\alpha, \beta, \gamma$; the novel PKCs, such as PKC $\delta, \varepsilon, \eta, \theta$, and the atypical PKCs, including PKC three subtypes of PKCs have conserved $\mathrm{C}$ terminus kinase domain, but the $\mathrm{N}$ terminus regulatory domain varies greatly among different subtypes. For example, the atypical PKCs lack the calcium binding domain and onehalf of the $\mathrm{C} 1$ homologous domain, therefore do not need diacylglycerol or calcium for their activation, but are dependent on lipid components, such as phosphatidylinositols (PIs) [5, 6].

The atypical PKCs, particularly the $\zeta$ isoform, have been implicated in numerous essential signaling events regulating tumorigenesis and cancer progression [7]. For 
example, earlier studies have shown that PKCל could bind with different protein regulators and adaptors to regulate the NF- $\mathrm{kB}$ pathway and control cell apoptosis [8-10]. PKCל is also reported to translocate to the nucleus under genotoxic conditions, where it regulates cell viability through the suppression of the apoptosis pathway and induces chemoresistance $[11,12]$. In addition, accumulating evidence have shown that PKC $\zeta$ plays a pivotal role in the regulation of cancer cell migration and invasion [13]. During breast cancer metastasis, EGF induces tumor cells intravasation from primary sites into circulation and SDF-1 mediates their extravasation to the secondary sites $[14,15]$. PKC $\zeta$ plays a regulatory role in both EGF and SDF-1 induced chemotaxis signaling pathways [16, 17]. In glioblastoma cells, pancreatic cancer cells and lung cancer cells, knockdown of PKC $\zeta$ dramatically reduces cell migration and invasion through regulation of the activities of numerous signaling molecules related with cytoskeleton rearrangement and cell adhesion, including cofilin, LIN-11, Isl1 and MEC-3 protein domain kinase (LIMK) and $\beta$-integrin $[18,19]$. However, the roles of $\mathrm{PKC \zeta}$ in neoplasia appear to be controversial. A number of clinical studies have shown that the expression levels of $\mathrm{PKC \zeta}$ vary among different types of tumor [7]. РКCל can inhibit Ras-induced tumorigenesis, and such tumor suppression effect is se-

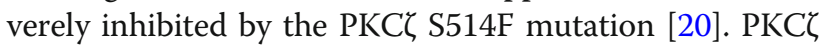
is also reported to phosphorylate c-Myc and suppress its activity, therefore inhibit tumorigenesis [21]. Furthermore, a recent study has implicated PKC $\zeta$ in the regulation of tumor metabolism. PKCद deficiency promotes the reprogramming of tumor metabolism to utilize glutamine through the serine biosynthetic pathway in the absence of glucose [22]. These evidence have shown that PKC $\zeta$ is involved in majority of the cancer hallmarks and modulate tumorigenesis through coordinating diverse molecular pathways. However, whether PKCל is a pro- or anti-neoplastic protein is still under debate, and further investigation is required to clarify its roles in different tumors.

The molecular mechanism by which PKCל participates in the regulation of cancer biology is largely dependent on its dynamic interactions with other proteins. For example, it has been reported that PKC $\zeta$ can modulate the NF- $\mathrm{kB}$ signaling pathway through interaction with p62 and par-4 $[10,23,24]$. Our previous study shows that rictor, a component of the mTOR complex 2, can bind with PKC $\zeta$ and mediates PKC $\zeta$ dependent breast cancer metastasis [25]. Therefore, to establish the interactome of $\mathrm{PKC \zeta}$ is crucial to understand the molecular mechanism whereby $\mathrm{PKC}$ regulates these signaling events.

In this study, proteomics and bioinformatics analyses were combined to establish a protein-protein interaction (PPI) network associated with PKCל. Proteins complexes associated with $\mathrm{PKC}$ from human breast cancer cell line MDA-MB-231 were purified with co-immunoprecipitation and analyzed by LC-MS/MS for protein identification. Two biological replicates and two technical replicates were analyzed. The observed proteins were filtered using the CRAPome database to eliminate the potential false positive identifications. For bioinformatics analysis, РКC $\zeta$ was searched against the STRING PPI database. The proteomics identification and database search results were combined for network construction. A PKC $\zeta$ centered PPI network was constructed, providing a more complete picture regarding the biological roles of $\mathrm{PKC \zeta}$ in the regulation of cancer hallmarks. Furthermore, molecular and cellular biology assays, such as immunofluorescence, coimmunoprecipitation (Co-IP), Western blotting, and cell migration assay were performed to study the biological implications of the interaction between $\mathrm{PKC} \zeta$ and protein phosphatase 2 catalytic subunit alpha (PPP2CA).

\section{Methods \\ Antibodies and reagents}

Mouse monoclonal antibody against Flag, anti-Flag antibody conjugated agarose beads, dithiothreitol (DTT), iodoacetamide (IAA) were from Sigma-Aldrich (St. Louis, MO, USA). Mouse monoclonal antibody against $\beta$-actin was from Santa Cruz (Santa Cruz, CA, USA). Mouse monoclonal antibody against $\mathrm{PKC \zeta}$ and rabbit polyclonal antibody against PPP2CA were from Cell Signaling Technology (Danvers, MA, USA). Lipofectamine 2000, BCA reagents, and Protein G agarose beads were purchased from Invitrogen. Enhanced chemiluminescence reagents were from Pierce Biotechnology. Protease Inhibitor Cocktail tablets were from Roche Diagnostics (Indianapolis, IN, USA). Sequencing grade modified trypsin was purchased from Promega (Madison, WI, USA). LC-MS grade water and acetonitrile were bought from Merck (White-house Station, NJ, USA).

\section{Clinical sample analysis}

The expression data of PKC $\zeta$ and PPP2CA in breast cancer was obtained through the cBio Cancer Genomics Portal (http://cbioportal.org), an open platform for exploring multidimensional cancer genomics data (TCGA) [26]. For survival curve analysis, the median mRNA expression level of PKC $\zeta$ was used as a cut-off value to divide the data into two groups. The survival curves of the high-expression and low-expression groups were compared using the log-rank tests.

\section{Cell culture, plasmid and transfection}

Human breast cancer cell line MDA-MB-231 and MCF7 were obtained from American Type Culture Collection. Cells were cultured in DMEM supplemented with 
$10 \%$ fetal bovine serum and $1 \%$ glutamine Pen-Strep solution at $37{ }^{\circ} \mathrm{C}$ and $5 \% \mathrm{CO}_{2}$.

Flag-PKC $\zeta$ was amplified by PCR and cloned into vector pcDNA 3.1. The Flag-PKC $\zeta$ plasmids were then transfected into MDA-MB-231 using Lipofectamine 2000 and Flag-PKC $\zeta$ stable cell line was established. The expression of Flag-PKC $\zeta$ fusion protein was confirmed by Western blotting with both anti-PKC $\zeta$ and anti-Flag antibodies.

For PPP2CA knockdown, three human PPP2CAsiRNA duplexes were designed and synthesized by RiboBio (Guangzhou, China). Non-targeting siRNA was also synthesized by RiboBio and used as negative control. The siRNAs was transfected into the cells by using X-tremeGENE siRNA Transfection Reagent (Roche, Indianapolis, IN, USA).

\section{Coimmunoprecipitation (Co-IP)}

Co-IP was performed to purify PKC and its interacting proteins. Briefly, cells were cultured to $80 \%-90 \%$ confluence and starved with serum free medium for $12 \mathrm{~h}$. Cellular proteins were extracted with lysis buffer (40 mM Tris, $120 \mathrm{mM} \mathrm{NaCl}, 1 \%$ Triton X-100, $1 \mathrm{mM}$ $\mathrm{NaF}, 1 \mathrm{mM} \mathrm{Na}_{3} \mathrm{VO}_{4}$ ) supplemented with protease inhibitor cocktail. Total protein concentration of the extract was measured with BCA assay. The cell extracts were precleared with protein $\mathrm{G}$ agarose beads, and then PKC $\zeta$ and its interacting proteins were isolated with anti-Flag antibody conjugated agarose beads, followed by Western blotting or mass spectrometric analysis.

\section{SDS-PAGE and western blotting}

Proteins were eluted from the agarose beads by incubation with the SDS-PAGE loading buffer in boiling water bath for $10 \mathrm{~min}$. For Western blotting, proteins separated by SDS-PAGE were transferred onto polyvinylidene fluoride membranes using a wet electro-blotter. The membranes were incubated with primary antibodies at $4{ }^{\circ} \mathrm{C}$ overnight, and followed by incubation with secondary antibodies at room temperature for $1 \mathrm{~h}$. Bound antibodies were detected by the ECL immumoblotting detection reagent.

\section{Proteolysis and mass spectrometric analysis}

PKCל interacting proteins were eluted from agarose beads with $6 \mathrm{M}$ urea in $25 \mathrm{mM}$ ammonium bicarbonate buffer, $\mathrm{pH}$ 8. The samples were reduced by incubating with $10 \mathrm{mM} \mathrm{DTT}$ at $37^{\circ} \mathrm{C}$ for $1 \mathrm{~h}$. The reduced proteins were alkylated for $1 \mathrm{~h}$ in darkness with $40 \mathrm{mM}$ iodoacetamide. The alkylation reaction was quenched by adding DTT to a final concentration of $50 \mathrm{mM}$. The urea in the solution was exchanged to $25 \mathrm{mM}$ ammonium bicarbonate buffer by centrifugation using $3 \mathrm{kDa}$ ultrafiltration devices (Millipore). Next, trypsin was added at a 50:1 protein to trypsin mass ratio, and the samples were incubated at $37^{\circ} \mathrm{C}$ overnight for the digestion to complete.

A nanoelectrospray ionization (nESI) LTQ XL linear ion trap mass spectrometer (Thermo Electron Corp) coupled with nanoLC system was used for protein identification. Two biological replicates and two technical replicates were analyzed. The LTQ mass spectrometer was operated in a data-dependent mode in which an initial MS scan recorded the mass range of $\mathrm{m} / \mathrm{z} 400-2000$, and the ten most abundant ions were automatically selected for CAD fragmentation. The spray voltage was set as $2.5 \mathrm{kV}$. The normalized collision energy was set at $35 \%$ for MS/MS. Raw LTQ data was searched against the IPI human protein database using SEQUEST algorithm embedded in the Protein Discoverer 1.3 Software (Thermo Electron Corp). The following parameters were applied during the database search: 1 Da precursor mass error tolerance, $1 \mathrm{Da}$ fragment mass error tolerance, static modifications of carbamido methylation for all cysteine residues and oxidation modifications of methionine residues. One missed cleavage site of trypsin was allowed. A reversed database was searched to evaluate the level of false discovery rate (FDR). FDR $<0.05$ was used as filtering criteria for proteins with multiple tryptic peptides, and FDR $<0.01$ was used for proteins identified with single tryptic peptide. Proteins with shared tryptic peptides were grouped and treated as one.

\section{Bioinformatics analysis}

The CRAPome database is a web-accessible (http:// www.crapome.org/) repository of negative-control AP-MS experiments. To eliminate potential false positive identifications, proteins identified using LC-MS/MS were uploaded to the CRAPome database and score $\geq 20$ was set as threshold for false positives. Known PPI information was obtained from both literature and several public PPI databases, including STRING (http://string90.embl.de/), Biogrid (http:// thebiogrid.org/), MINT (http://mint.bio.uniroma2.it/), IntAct (http://www.ebi.ac.uk/intact/) and HPRD (http:// www.hprd.org/). The PPI data obtained through database search and mass spectrometric analysis was then integrated in Excel and imported into Cytoscape v2.8.3 (http:// www.cytoscape.org/) for network visualization. Gene ontology annotation was conducted by the PANTHER database program (http://www.pantherdb.org/) [27]. Pathway analysis was performed using DAVID (https://david.ncifcrf.gov/).

The interactome network analysis was conducted using the Systems Biology and Evolution MATLAB Toolbox (SBEToolbox) and Cytoscape. Several characteristic properties of the constructed network were computed, including node-specific degree $\mathrm{k}$, clustering coefficient, and small-world index. The power-law degree distributions and adjacency matrices of the networks were generated using MATLAB. The rich-club coefficient is computed as 
described by others [28, 29]. Briefly, we generated 1000 comparable random networks with equal size and the same degree distribution by rewiring to calculate the normalized rich-club coefficient. When the normalized richclub coefficient is greater than 1 , it indicates the rich-club organization in the network is significant.

\section{Immunofluorescence}

Cells were cultured on six-well chamber slides. At the time of harvest, cells were fixed with $4 \%$ paraformaldehyde and then permeabilized with $0.01 \%$ Triton X-100 for $10 \mathrm{~min}$. The cells were incubated with primary antibodies at $4{ }^{\circ} \mathrm{C}$ overnight, followed by staining with Alexa Fluor 488 and 546-conjugated secondary antibodies for $1 \mathrm{~h}$ at room temperature. All samples were treated with 4',6-diamidino-2-phenylindole (DAPI) dye for nuclear staining. A Nikon C2 Plus confocal microscope was applied for imaging.

\section{Quantitative reverse transcription PCR (qRT-PCR)}

Total RNA was isolated by Trizol reagent (Life Technologies, Carlsbad, CA, USA). Reverse transcription reactions were performed with $5 \mu \mathrm{g}$ of total RNA using a FastQuant RT kit (TIANGEN, Beijing, China) according to the manufacturer's protocols. The cDNA was subjected to quantitative real-time PCR (qRT-PCR) using the SYBR Green PCR Kit (TIANGEN, China) and the assay was performed on an ABI PRISM 7500 Sequence Detector. Expression data were uniformly normalized to GAPDH as an internal control, and the relative expression levels were evaluated using the $2^{-\Delta \Delta C t}$ method. The primer sequences for PPP2CA were 5'-GAT CTT CTG TCT ACA TGG TGG TCT C-3' (Forward) and 5'-ACA CAT TGG ACC CTC ATG GGG AA-3' (Reverse). GAPDH was used as an internal control (forward: 5'-TGC ACC ACC AAC TGC TTA GC-3'; reverse: 5'-GGC ATG GAC TGT GGT CAT GAG-3').

\section{Cell migration assay}

In wound healing assay, MDA-MD-231 cells were seeded in 6-well plates and grown until 80-90\% confluence. The cells were scratched with a pipette tip in the middle of the plate, washed with PBS to remove the detached cells and incubated in a medium containing $1 \%$ FBS. The wound closure was monitored microscopically at different time-points and photographed at 0 and 24 h respectively.

\section{Statistical analysis}

SPSS version 17.0 software were performed for statistical analyses and Prism version 5.0 (GraphPad) were used to plot to show mean and standard deviation (SD). Student's $t$ test was performed for comparison. All statistical tests were two-sided and $P$ values were considered statistically significant for $P \leq 0.05$.

\section{Results}

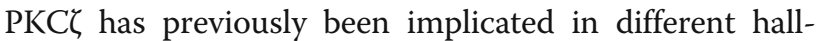
marks of cancer [7]. We first analyzed the expression of PKC $\zeta$ in breast cancer by searching the TCGA database. Survival analysis based on RNA sequencing data from 1445 patients in TCGA database showed that the overexpression of $\mathrm{PKC \zeta}$ is associated with poor prognosis $(P=0.0011$, Additional file 1: Figure S1A). To better understand the biological roles of PKC $\zeta$ in the diverse signaling pathways regulating cancer, proteomics and bioinformatics analyses were combined to establish a PPI network associated with PKCఢ. As shown in Fig. 1, PKC $\zeta$ interacting proteins were isolated with Co-IP and analyzed by LC-MS/MS for protein identification. The observed proteins were filtered using the CRAPome database to eliminate the potential false positives. The MS identification results were combined with PPI database search to construct the PKC interactome network. The $\mathrm{PKC} \zeta$ interacting proteins were further analyzed using the PANTHER database and the DAVID database.

\section{Characterization of $\mathrm{PKC}$ interacting proteins}

Firstly, CoIP-MS was employed to identify PKC $\zeta$ interacting proteins. MDA-MD-231 breast cancer cells were transfected with Flag-PKC $\zeta$ fusion protein and stable clones were cultured. As shown in Fig. 2a, Western

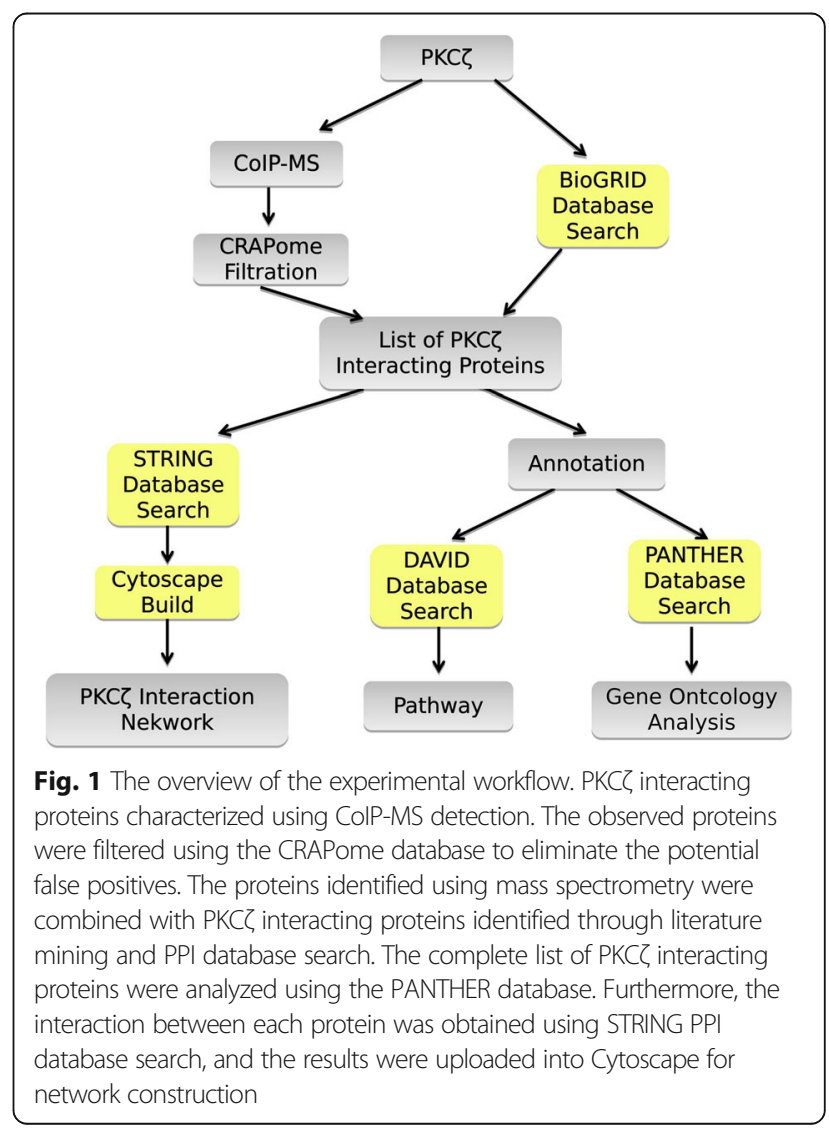


blotting showed that the stable clone cells successfully expressed Flag-PKC lated using anti-Flag antibody from the Flag-PKC cells. PKC $\zeta$ was enriched in the immunoprecipitates as detected with Western blotting and SDS-PAGE (Fig. 2). Using LCMS/MS analysis, 233 proteins were detected in the Flag immunoprecipitates. After CRAPome filtration, 106 proteins were identified as potential PKC $\mathrm{P}$ interacting proteins (Additional file 1: Table S1). Some of these proteins are known PKC interacting proteins, such as sequestosome 1/p62 (SQSTM1) and complement component C1qbinding protein (C1QBP). PPI Databases search was also performed to achieve comprehensive identification of PKC $\zeta$ interacting proteins. Combining literature mining and searching through several PPI databases, including BioGrid, InACT, STRING, MINT and HPRD, we were able to obtain 77 PKC $\zeta$ interacting proteins (Additional file 1: Table S2). This set of data included some of the well known $\mathrm{PKC}$ interacting proteins, such as AKT and several other isoforms of PKCs.

\section{Gene ontology analysis of the PKC interacting proteins}

The combined PKC $\zeta$ interacting proteins were further analyzed for gene ontology annotation. As shown in Fig. 3a, molecular function analysis revealed that most of the identified proteins were related with protein binding (38.4\%), catalytic activity (28.6\%), structural molecule activity (11.4\%), nucleic acid binding transcription regulation activity (5.4\%), and enzyme regulator activity
(4.3\%). As shown in Fig. 3b, biological process analysis showed that PKC $\zeta$ interacting proteins are associated with metabolic process $(24.6 \%)$, cellular process $(21.3 \%)$, biological regulation (10.9\%), response to stimulus (8.6\%), developmental process (7.9\%), cellular component organization or biogenesis (7.1\%), and immune system process (5.6\%). Cellular component analysis showed that these non-specific binding proteins were from various regions of the cell, such as cell part (42.1\%), organelle (21.1\%) and macromolecular complex (20.0\%), and membrane (9.5\%) (Fig. 3c). As shown in Fig. 3d, the major protein classes included nucleic acid binding $(16.6 \%)$, transferase $(10.2 \%)$, kinase $(8.3 \%)$, chaperone (7.8\%), calcium binding protein $(6.8 \%)$, and cytoskeletal proteins (5.9\%).

Associated signaling pathways were analyzed using DAVID, and the related signaling pathways with $P$ value $\leq 0.01$ were shown in Fig. 3e and Additional file 1: Table S3. The top related signaling pathway (with 19 associated proteins) is the EGF signaling pathway. The FGF and PDGF signaling pathways are also relevant, and many proteins in these two pathways overlap with the EGF pathway. The next significantly related signaling pathway is the apoptosis pathway. PKCל interacts with 14 proteins from the apoptosis pathway, such as the inhibitor of nuclear factor kappa-B kinase subunit beta (IKBKB). The inflammation mediated by chemokine and cytokine signaling pathway is also highly relevant. The pathway analysis results are highly consistent with the known functions of PKCद.

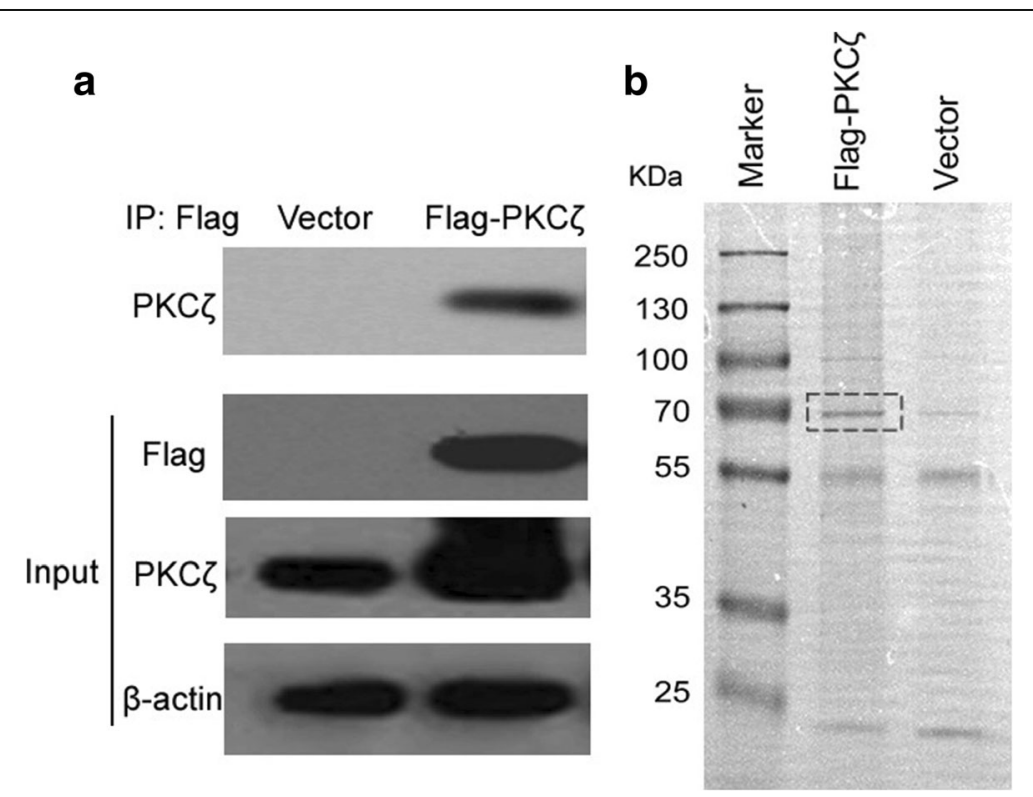

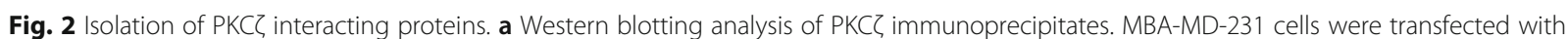

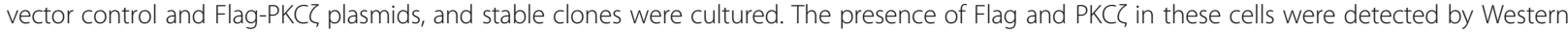
blotting. Co-IP was performed using anti-Flag conjugated beads. b SDS-PAGE separation of the Flag-PKC immunoprecipitates. The gel was visualized using silver staining 

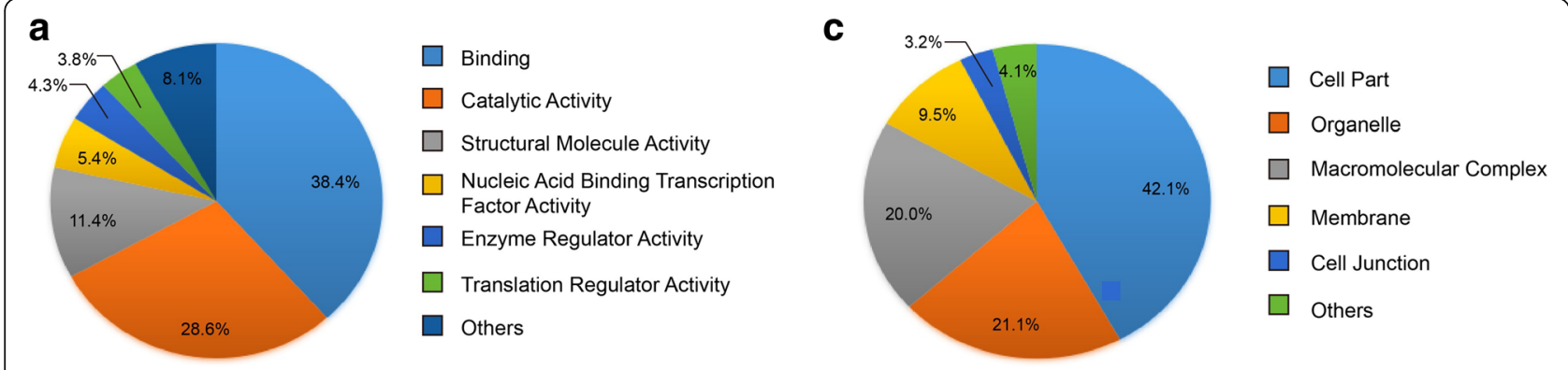

b

d
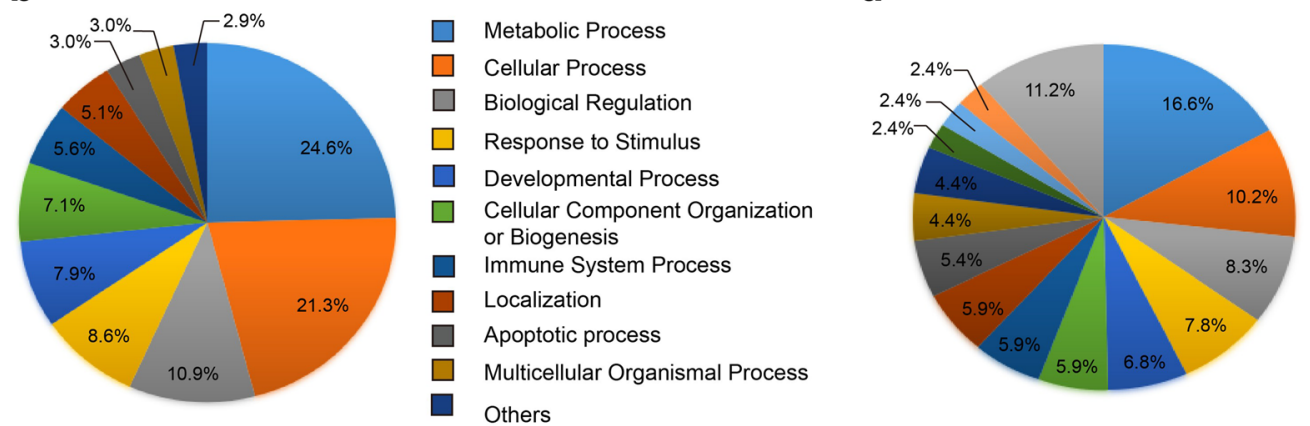

Nucleic Acid Binding

$\square$ Transferase

$\square$ Kinase

$\square$ Chaperone

$\square$ Calcium-Binding Protein

$\square$ Cytoskeletal Protein

$\square$ Enzyme Modulator

$\square$ Transcription Factor

$\square$ Transfer/Carrier Protein

$\square$ Signaling Molecule

$\square$ Hydrolase

$\square$ Transporter

$\square$ Ligase

$\square$ Phosphatase

e

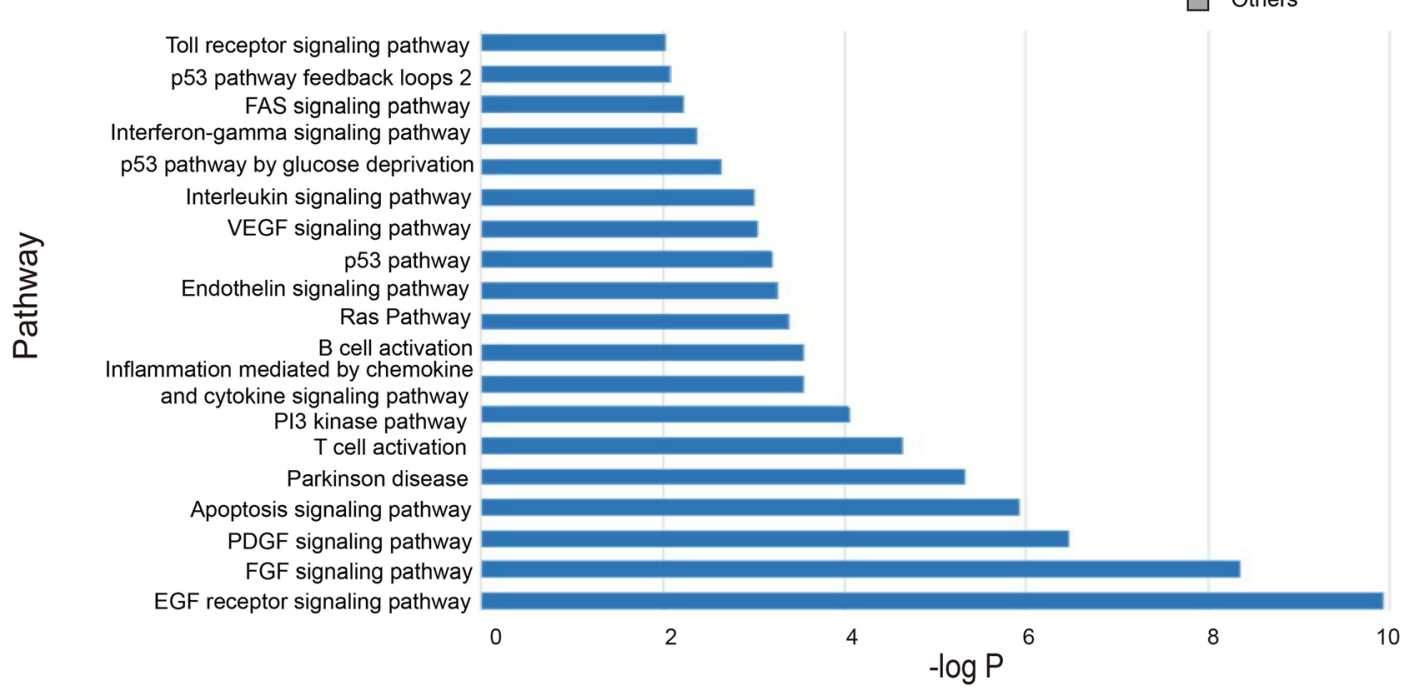

Fig. 3 Diagram showing the assignment of gene ontology terms to the identified PKC $\zeta$ interacting proteins: (a) molecular functions. b biological process. c cellular component. $\mathbf{d}$ protein classes. Analysis was performed using the PANTHER database program (www.pantherdb.org). e The signaling pathways associated with the PKCZ interacting proteins

\section{Construction and analysis of the PKCZ interactome network}

The PKC interacting proteins obtained through mass spectrometry analysis and database mining were searched against the STRING database for interaction information and imported to Cytoscape for network construction. As shown in Fig. 4, a highly connected network composed of 183 proteins and 1225 connections was mapped. About half of the mapped proteins were from data search and literature, and half of the proteins were identified with mass spectrometry analysis. Only 12 proteins were observed using both methods, including C1QBP, SQSTM1, JAK1, LLGL1, etc.

Most of the known PKC interacting proteins from literature and databases were identified using Western blotting or yeast two hybrid. These methods may scope different types of proteins compared to mass spectrometric analysis. 


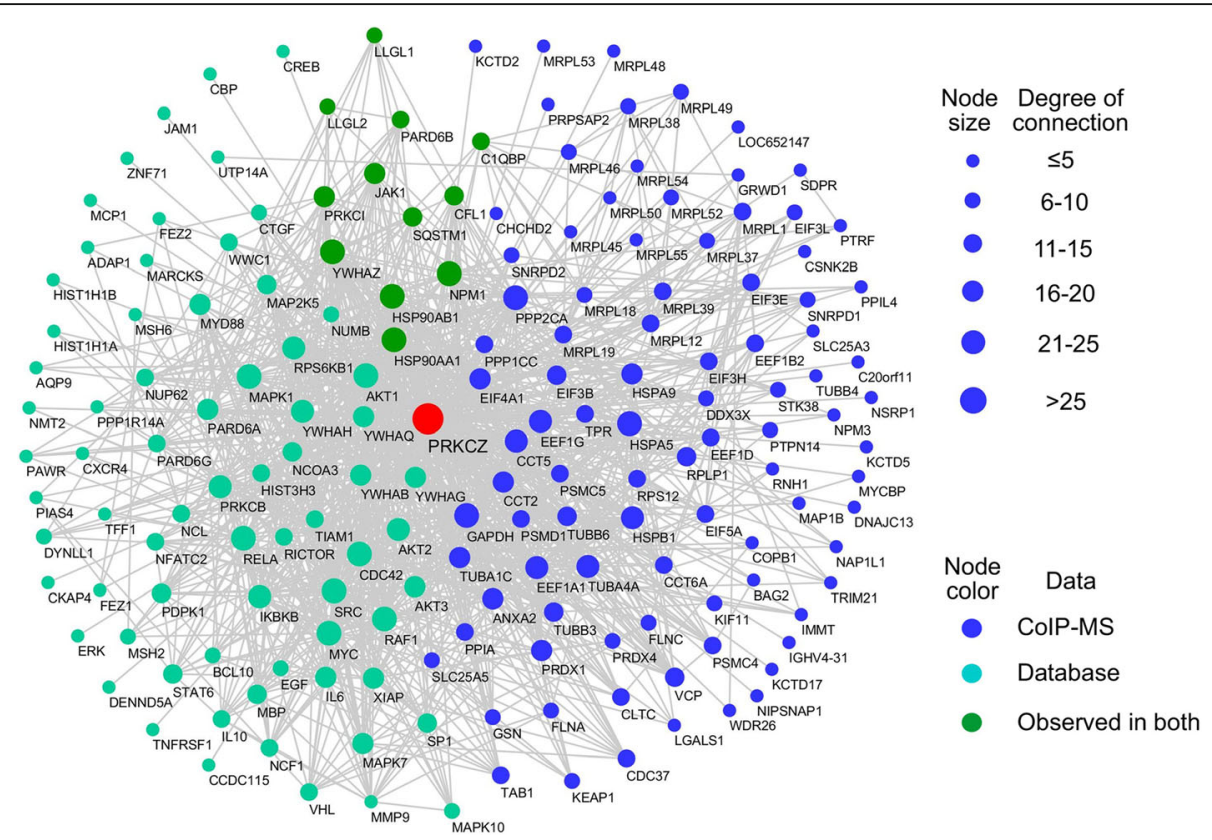

Fig. 4 PPI network of the PKC interacting proteins. PKC interacting proteins (from Additional file 1: Table S1) and their interactions are shown as nodes and edges. Proteins identified using mass spectrometry and database search were shown in different colors as indicated in the graph. Node size reflects the interaction degree as indicated. The network was constructed using Cytoscape

As shown in Fig. 4, some of these proteins are highly connected in the constructed network (degree of connection was indicated with node size). To further understand the properties of the constructed PKC $\mathrm{Pin}$ teractome network, a rich-club analysis was conducted. A rich club is a set of high-degree nodes that are more densely interconnected than predicted by the node degrees alone [29]. The rich-club nodes may form a hub that is used by the other components in the network to influence each other. As shown in Fig. 5a, the PKC interactome network exhibits a power-law degree distribution consistent with being a scale-free network. The results suggest that the frequency of nodes negatively correlates with the connection degree indicating a few number of nodes have the majority of the interactions in the network and therefore may form a connection hub. Further analysis of the network clustering coefficient showed that the PKC interactome network has relatively higher clustering coefficient and higher smallworld index as compared to the random networks (Fig. $5 b)$. In addition, the presence of a rich-club organization within the PKC interactome network was characterized. To investigate the significance of the discovered richclub, the rich-club coefficient of the PKC $\zeta$ interactome network was compared to that of 1000 randomly generated networks with similar degree distribution. The normalized rich-club coefficient reveals the presence of a significant rich-club between degrees 11 and 175 and a peak at degree 29 (Fig. 5c). The sub-network of nodes with degrees corresponding to the highest normalized rich-club coefficient (above 1.2) was shown in Fig. 5d. This core network contained 20 nodes and 141 edges. The rich-club network includes some of the most well known PKC $\zeta$ interacting proteins, such as AKT1, IKBKB, MAPK1, etc. These proteins may play a more influential role within the overall interactome network of PKCद.

\section{The interaction between PPP2CA and PKC}

PPP2CA is one of the four newly identified PKC interacting proteins that present in the rich-club core network. PPP2CA is a component of the protein phosphatase 2A (PP2A), an important and ubiquitously expressed serine threonine phosphatase that regulates many cellular processes by dephosphorylating critical cellular molecules like AKT, P53, c-Myc and $\beta$-catenin $[30,31]$. The PP2A hetero-trimer consists of a catalytic core comprised of the catalytic A and $C$ subunits as well as a regulatory $B$ subunit that controls substrate specificity and cellular localization [32]. The interaction between PPP2CA and PKCद may be very important for their functions in different biological processes. Therefore, we sought to validate their interaction. To investigate the correlation of these two studied proteins, we analyzed their expressions in 1145 breast cancer samples from TCGA. As shown in Fig. 6a, PPP2CA and PKC regulated in breast cancer tumor tissues. Co-IP and 


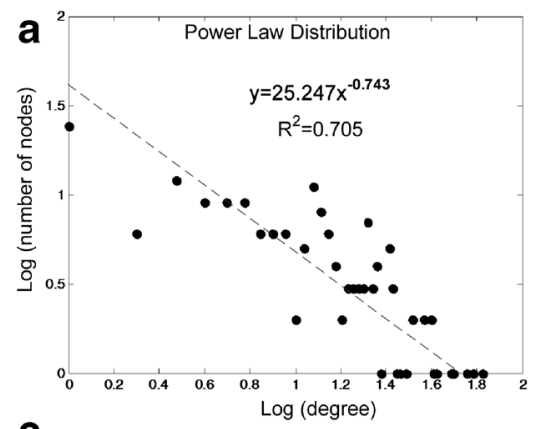

C

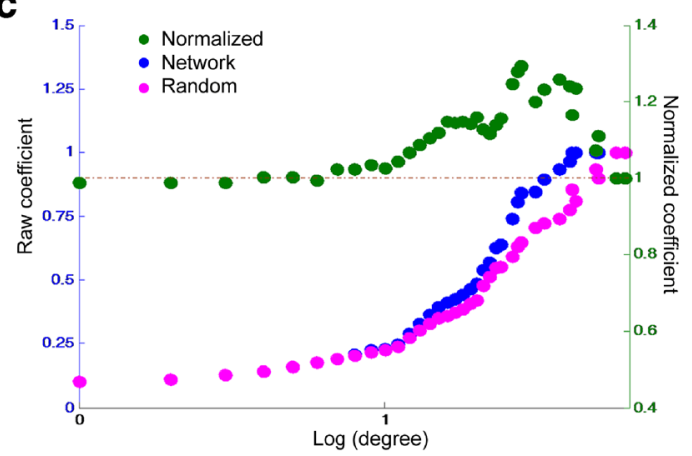

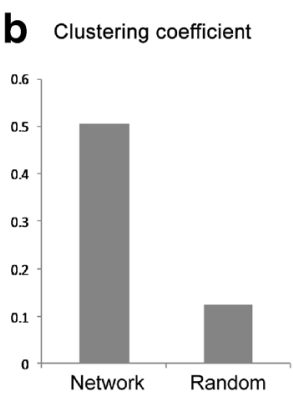

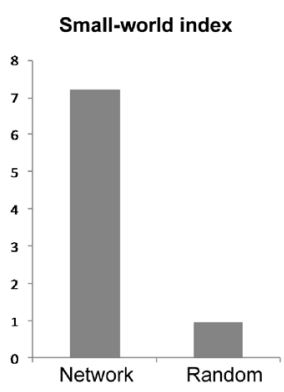

d

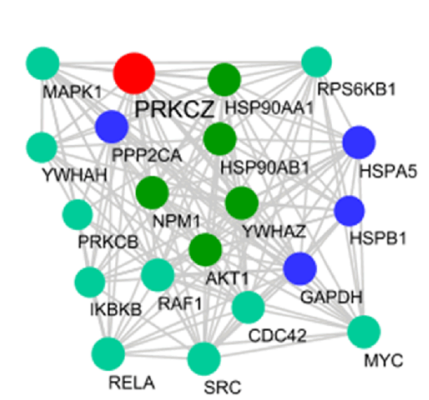

Fig. 5 Properties of the PKC PPI network. a Power-law distribution curve of the PKC network shows a negative correlation between the number of nodes ( $y$ axis) and the node-specific degree ( $x$ axis), Pearson correlation coefficient $\rho=-0.856$. $\mathbf{b}$ Identification of the small-world organization within the PKC PPI network. Clustering coefficient of constructed network was significantly higher as compared with that of the random networks. The small-world coefficient was 7.2. c Raw rich-club coefficient of the constructed PKC PPI network (blue) and random network (red) plotted against the left vertical axis. Normalized rich-club coefficient for the network (green) plotted against the right vertical axis. $\mathbf{d}$ The sub-network of the rich-club organization within the PKC network. Proteins are indicated with circles with different colors as used in Fig. 5, and the interactions between proteins are indicated with grey edges

Western blotting showed that PPP2CA could indeed bind to $P K C \zeta$ in two types of human breast cancer cell line, including MDA-MB-231 and MCF-7 (Fig. 6b). In addition, immunefluorescence showed that these two proteins both localized in the cytoplasm (Fig. 6c). Our previous study has shown that PKC is a key regulatory molecule that promotes cell migration and breast cancer metastasis [16]. To understand the biological impact of the interaction between PKC $\zeta$ and PPP2CA, we knocked down PPP2CA from MDA-MB-231 cells and investigated its effect on cell migration. Three siRNAs were used to silence PPP2CA from MDA-MB-231 cells. qRT-PCR and Western blotting showed that sequence \#2 and \#3 could efficiently knocked down the levels of PPP2CA from the cells. Results from cell migration assay showed that down-regulation of PPP2CA increased cell migration in MDA-MB-231 cells (Fig. 6d). Taken together, the results implicated that PPP2CA may affect breast cancer cell migration through interacting with PKCद.

\section{Discussion}

In this study, we combined proteomics and bioinformatics analysis to construct a comprehensive $\mathrm{PKC} \zeta$ interactome network consisting of 178 proteins and 1225 connections. This map is important for further understanding the complicated roles $\mathrm{PKC} \zeta$ plays in the diverse biological processes regulating cancer. Previous studies have suggested that the activation of PKC $\zeta$ is controlled by phosphoinositide 3-kinase (PI3K) and PDK1 [33, 34], which are major downstream effectors of receptor tyrosine kinases, including EGFR, PDGFR, FGFR, VEGFR, etc. Consistent with these reports, our results showed that the top 3 related signaling pathways are EGF, FGF and PDGF pathways. It is well known that activated receptor tyrosine kinases regulate cellular processes through two major pathways: PI3K/Akt and Ras/ MAPK signaling. In this study, we found that several PKC $\zeta$ binding proteins are associated with these two cellular signaling pathways. In addition, the results showed that multiple PKC binding proteins are associated with chemokine and cytokine signaling pathways. In agreement with these observations, previous studies have shown that PKCद is involved in the regulation of directional cell migration, such as chemotaxis, which plays a critical role in cancer cell invasion and metastasis [35-37]. Intensive studies indicate that PKC $\zeta$ is a key mediator of EGF-induced chemotaxis and is required for cancer cell metastasis $[1,38-40]$. Together, this study provides a detailed map of the PKC $\zeta$ centered PPIs and their coordination that regulate these pathways. 


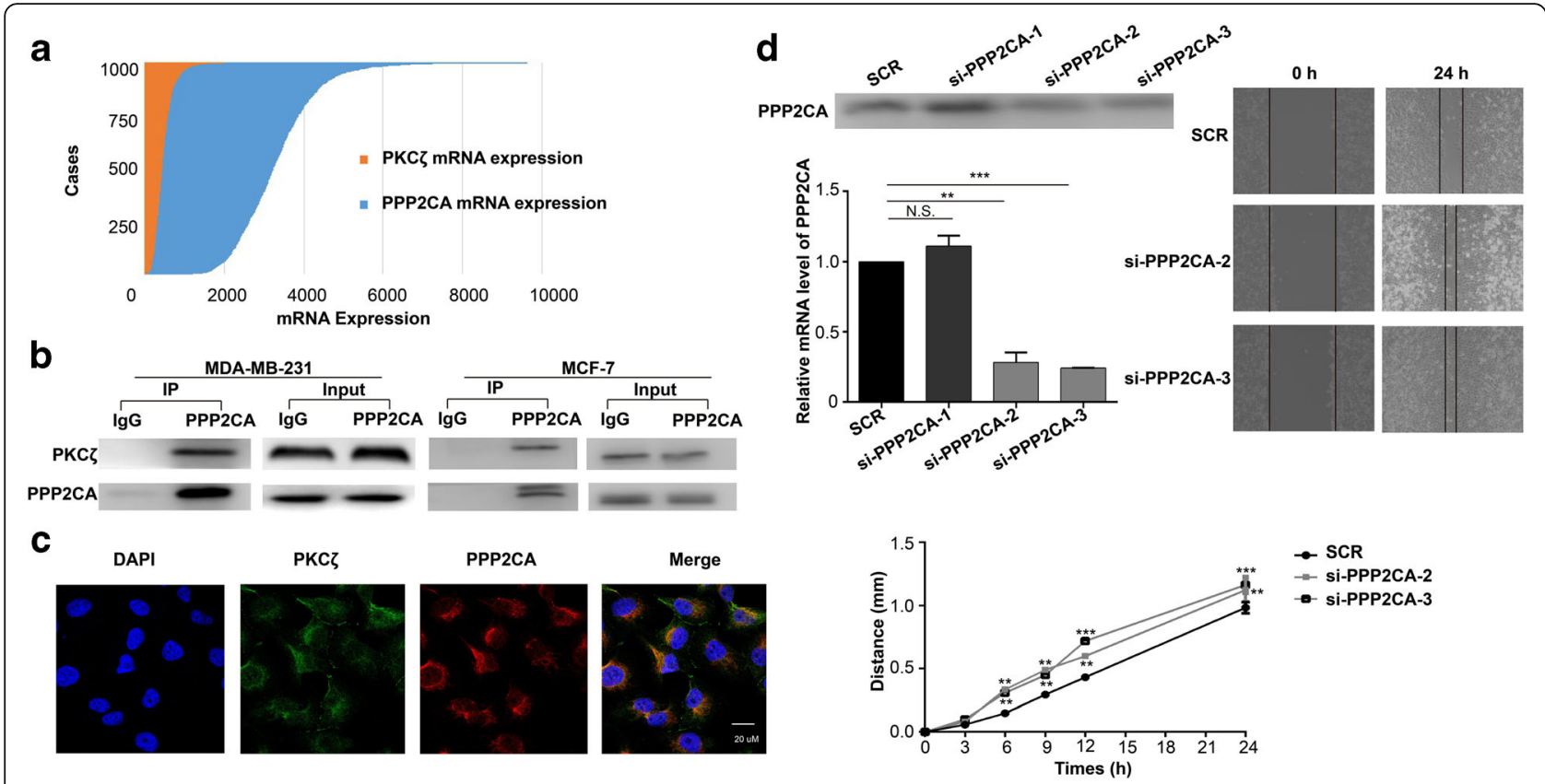

Fig. 6 The interaction between PKC and PPP2CA. a The mRNA levels of PPP2CA and PKC $\zeta$ in breast cancer samples from the TCGA database. $\mathbf{b}$ Western blotting analysis of the PPP2CA immunoprecipitates from MDA-MB-231 and MCF-7 cells. Co-IP was performed using anti-PPP2CA antibody conjugated protein $\mathrm{G}$ agarose beads. Normal lgG was used as control. c Immunofluorescence analysis of PKC and PPP2CA in MDA-MB231 cells. Cell nuclei were stained with DAPI. d PPP2CA knockdown in MDA-MB-231 cell enhanced cell migration. The efficiency of PPP2CA knockdown was examined by qRT-PCR and Western blotting. Bar; mean; error bar: SD $\left({ }^{*} P<0.05,{ }^{* *} P<0.01,{ }^{* * *} P<0.0001\right.$, by student's $t$-test)

The core network achieved through rich-club analysis indicated that 20 proteins are highly connected with PKCל, such as AKT, MAPK1, IKBKB, MYC, etc. These proteins may play a more important role in the $\mathrm{PKC \zeta}$ network. The direct interaction between PKC $\zeta$ and AKT2 has been implicated in chemotaxis, and AKT2 directly mediates EGF-induced chemotactic signaling pathways through

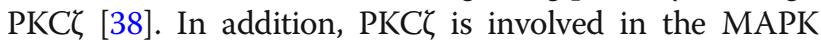
cascade. Through participating in TNF-dependent transactivation of NF-kappa-B through phosphorylating and activating IKBKB kinase, PKC $\zeta$ leads to degradation of NF- $k B$ inhibitors [6]. Furthermore, decreased phosphoryl-

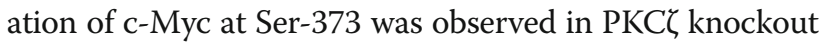
tumors, suggesting $\mathrm{PKC \zeta}$ is a critical regulator of $\mathrm{c}-\mathrm{Myc}$ [21]. Investigating other proteins mapped in the rich club network and their interactions will be helpful to further elucidate the functions of $\mathrm{PKC} \zeta$ in tumorigenesis and cancer metastasis.

In this study, we validated PPP2CA as a novel PKC interacting protein. PPP2CA gene encodes the catalytic subunit $\mathrm{C}$ of PP2A, which is one of the four major Ser/ Thr phosphatases [41]. PP2A plays critical roles in diverse cellular processes, such as cell proliferation [42], signal transduction [43] and apoptosis [44]. Some of these functions overlap with PKCद. Intriguingly, the interaction we observed here is between a phosphatase and a kinase, and it has been reported that the activations of both PPP2CA and PKC $\zeta$ depend on their phosphorylations. Therefore, it is very likely that they could regulate the activities of each other through phosphorylation and de-phosphorylation. It would be interesting to further investigate the biological functions of this interaction and to reveal the underlying molecular mechanism.

\section{Conclusions}

In this study, the PPI network of PKC $\zeta$ containing 178 nodes and 1225 connections was constructed through combining proteomics and bioinformatics analyses. A comprehensive gene ontology and pathway analysis was performed on the PKC $\zeta$ interacting proteins. The results suggest that PKC $\zeta$ may regulate multiple cellular processes through coordinating diverse signaling pathways related with cancer. This study provides a more complete picture regarding the biological roles of $\mathrm{PKC} \zeta$ in both cancer regulation and other aspects of cellular biology.

\section{Additional file}

Additional file 1: Supplemental information. (DOC 78 kb)

\section{Abbreviations}

C1QBP: Complement component C1qbinding protein; Co-IP:

Co-immunoprecipitation; DAPI: 4',6-diamidino-2-phenylindole;

DTT: Dithiothreitol; FDR: False discovery rate; GO: Gene ontology;

IAA: lodoacetamide; IKBKB: Inhibitor of nuclear factor kappa-B kinase 
subunit beta; LIMK: LIM domain kinase; nESI: Nanoelectrospray ionization; PI3K: Phosphoinositide 3-kinase; Pls: Phosphatidylinositols: PKC: Protein kinase C; PKC ${ }_{\text {: }}$ Protein kinase C $\zeta_{\text {; }}$ PP2A: Protein phosphatase 2A; PPI: Protein-protein interaction; PPP2CA: Protein phosphatase 2 catalytic subunit alpha; qRT-PCR: Quantitative reverse transcription PCR; SQSTM1: Sequestosome 1/p62

\section{Acknowledgments}

Not applicable.

\section{Funding}

This work was supported by grants from National Natural Science Foundation of China (21575103, 61376082, 31671421, and 81472683), 863 program (2015AA020403); National Key Research and Development Program (2016YFC0900100)

\section{Availability of data and materials}

Processed data included within Additional file 1: Tables S1-S3 and Figure S1A.

\section{Authors' contributions}

$\mathrm{CH}$ carried out the mass spectrometric analysis and clinical sample analysis. $\mathrm{CH}$ and $\mathrm{YL}$ performed cell experiments. $\mathrm{CH}$ and MD performed the bioinformatics analysis. GQ, NZ, and RC supervised the study and wrote the manuscript. All authors read and approved the final manuscript.

\section{Ethics approval and consent to participate}

Not applicable.

\section{Consent for publication}

Not applicable.

\section{Competing interests}

The authors declared that they have no conflicts of interest to this work. We declare that we do not have any commercial or associative interest that represents a conflict of interest in connection with the work submitted.

\section{Publisher's Note}

Springer Nature remains neutral with regard to jurisdictional claims in published maps and institutional affiliations.

\section{Author details}

${ }^{1}$ Tianjin Medical University Cancer Institute and Hospital, National Clinical Research Center for Cancer, Key Laboratory of Cancer Prevention and Therapy, Tianjin's Clinical Research Center for Cancer, Tianjin 300070, China. ${ }^{2}$ Department of Genetics, School of Basic Medical Sciences, Tianjin Medical University, Tianjin 300070, China. ${ }^{3}$ School of Microelectronics, Tianjin University, Tianjin 300072, China.

\section{Received: 20 October 2017 Accepted: 11 February 2018}

\section{Published online: 26 February 2018}

\section{References}

1. Lipp P, Reither G, Protein Kinase C. The "masters" of calcium and lipid. CSH Perspect in. Biol. 2011;3:322-30

2. Newton $A C$. Regulation of the $A B C$ kinases by phosphorylation: protein kinase $C$ as a paradigm. Biochem J. 2003;370:361-71.

3. Xiao H, Liu M. Atypical protein kinase C in cell motility. Cell Mol Life Sci. 2013;70:3057-66.

4. Griner EM, Kazanietz MG. Protein kinase $C$ and other diacylglycerol effectors in cancer. Nat Rev Cancer. 2007;7:281-94.

5. Nakanishi $\mathrm{H}$, Brewer KA, Exton $\mathrm{JH}$, et al. Activation of the zeta isozyme of protein kinase C by phosphatidylinositol 3,4,5-trisphosphate. J Biol Chem. 1993;268:13-6.

6. Sun J. GPCR-CARMA3-NF-kappaB signaling axis: a novel drug target for cancer therapy. Clin Oncol. Cancer Res. 2010;7:159-68.

7. Rimessi A, Patergnani S, loannidi E, et al. Chemoresistance and cancer-related inflammation: two hallmarks of cancer connected by an atypical link. PKC了 Front Oncol. 2013;3:232.

8. Moscat J, Rennert P, Diazmeco MT, et al. PKC at the crossroad of NF-kB and Jak1/Stat6 signaling pathways. Cell Death Differ. 2006;13:702-11.
9. Diaz-Meco MT, Dominguez I, Sanz L, et al. PKCZ induces phosphorylation and inativation of IKB-a in vitro. EMBO J. 1994;13:2842-8.

10. Diazmeco MT, Municio MM, Frutos S, et al. The product of par-4, a gene induced during apoptosis, interacts selectively with the atypical isoforms of protein kinase C. Cell. 1996;86:777-86.

11. Mas VM, Hernandez H, Plo I, et al. Protein kinase C zeta mediated Raf-1/ extracellular-regulated kinase activation by daunorubicin. Blood. 2003;101: 1543-50.

12. Bezombes $C$, De TA, Apostolou A, et al. Overexpression of protein kinase $C$ zeta confers protection against antileukemic drugs by inhibiting the redoxdependent sphingomyelinase activation. Mol Pharmacol. 2002;62:1446-55.

13. Hirai T, Chida K. Protein kinase C (PKC)): activation mechanisms and cellular functions. J Biochem. 2003;133:1-7.

14. Wells A. Tumor invasion: role of growth factor-induced cell motility. Adv Cancer Res. 2000;78:31-110.

15. Teicher BA, Fricker SP. CXCL12 (SDF-1)/CXCR4 pathway in cancer. Clin Cancer Res. 2010;16:2927-31.

16. Sun $R$, Gao $P$, Chen $L$, et al. Protein kinase $C \zeta$ is required for epidermal growth factor-induced chemotaxis of human breast cancer cells. Cancer Res. 2005;65:1433-41.

17. Laudanna C, Mochly-Rosen D, Liron T, et al. Evidence of zeta protein kinase $\mathrm{C}$ involvement in polymorphonuclear neutrophil integrin-dependent adhesion and chemotaxis. J Biol Chem. 1998;273:30306-15.

18. Guo H, Gu F, Li W, et al. Reduction of protein kinase $\mathrm{C}$ zeta inhibits migration and invasion of human glioblastoma cells. J Neurochem. 2009;109:203-13.

19. Liu Y, Wang B, Wang J, et al. Down-regulation of PKCZ expression inhibits chemotaxis signal transduction in human lung cancer cells. Lung Cancer. 2009;63:210-8

20. Galvez AS, Duran ALJF, Pathrose $P$, et al. Protein kinase $C$ zeta represses the interleukin-6 promoter and impairs tumorigenesis in vivo. Mol Cell Bio. 2009;29:104-15.

21. Ji YK, Valencia T, Abu-Baker S, et al. C-Myc phosphorylation by PKC乙 represses prostate tumorigenesis. PNAS. 2013;110:6418-23.

22. Ma L, Tao Y, Duran A, et al. Control of nutrient stress-induced metabolic reprogramming by PKC in tumorigenesis. Cell. 2013;152:599-611.

23. Sanz $L$, Sanchez $P$, Lallena $M$, et al. The interaction of $p 62$ with RIP links the atypical PKCs to NF-KB activation. EMBO J. 1999;18:3044-53.

24. Chang S, Kim J H, Shin J. P62 forms a ternary complex with PKC zeta and PAR-4 and antagonizes PAR-4-induced PKC zeta inhibition. FEBS Lett. 2002; 510:57-61.

25. Zhang F, Zhang X, Li M, et al. mTOR complex component Rictor interacts with PKC zeta and regulates cancer cell metastasis. Cancer Res. 2010;70:9360-70.

26. Cerami E, Gao J, Dogrusoz U, et al. The cBio cancer genomics portal: an open platform for exploring multidimensional cancer genomics data. Cancer Discov. 2012;2:401-4.

27. Thomas PD, Kejariwal A, Campbell MJ, et al. PANTHER: a browsable database of gene products organized by biological function, using curated protein family and subfamily classification. Nucleic Acids Res. 2003;31:334-41.

28. Colizza V, Flammini A, Serrano MA, et al. Detecting rich-club ordering in complex networks. Nat Phys. 2006;2:110-5.

29. Alawieh A, Sabra Z, Sabra M, et al. A rich-club organization in brain ischemia protein interaction network. Sci Rep. 2015;5:1-13.

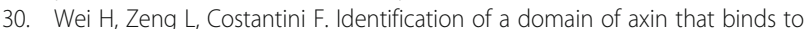
the serine/threonine protein phosphatase $2 \mathrm{~A}$ and a self-binding domain. J Bio Chem. 1999:274:3439-45.

31. Watkins GR, Wang N, Mazalouskas MD, Gomez RJ, Guthrie CR, Kraemer BC, Schweiger S, Spiller BW, Wadzinski BE. Monoubiquitination promotes calpain cleavage of the protein phosphatase 2A (PP2A) regulatory subunit a 4, altering PP2A stability and microtubule-associated protein phosphorylation. J Bio Chem. 2012;287:24207-15.

32. Dorien $\mathrm{H}$, Ward $\mathrm{S}$, Katleen $\mathrm{L}$, et al. The basic biology of PP2A in hematologic cells and malignancies. Front Oncol. 2014;4:347-58.

33. Lee JJ, Loh K, Yap YS. PI3K/Akt/mTOR inhibitors in breast cancer. Cancer Biol Med. 2015;12:342-54.

34. Good JAL, Ziegler WH, Parekh DB, et al. Protein kinase C isotypes controlled by phosphoinositide 3-kinase through the protein kinase PDK1. Science. 1998;281:2042-5.

35. Etiennemanneville S, Hall A. Cell polarity: Par6, aPKC and cytoskeletal crosstalk. Curr Opin Cell Biolgy. 2003;15:67-72. 
36. Etienne-Manneville S, Manneville JB, Nicholls S, et al. Cdc42 and Par6-PKC zeta regulate the spatially localized association of Dlg1 and APC to control cell polarization. J Cell Biol. 2005;170:895-901.

37. Pegtel DM, Ellenbroek SI, Mertens AE, et al. The par-Tiam1 complex controls persistent migration by stabilizing microtubule-dependent front-rear polarity. Curr Biol. 2007:17:1623-34.

38. Parsons M, Keppler MD, Kline A, et al. Site-directed perturbation of protein kinase C-integrin interaction blocks carcinoma cell chemotaxis. Mol Cell Biol. 2002;22:5897-911.

39. Giagulli C, Scarpini E, Ottoboni L, et al. RhoA and PKC zeta control distinct modalities of LFA-1 activation by chemokines: critical role of LFA-1 affinity triggering in ly5mphocyte in vivo homing. Immunity. 2004;20:25-35.

40. Litherland GJ, Elias MS, Wang $\mathrm{H}$, et al. Protein kinase $\mathrm{C}$ isoforms $\zeta$ and I mediate collagenase expression and cartilage destruction via STAT3- and ERK-dependent c-fos induction. J Biol Chem. 2010;285:22414-25.

41. Ruvolo PP. The broken "off" switch in cancer signaling: PP2A as a regulator of tumorigenesis, drug resistance, and immune surveillance. BBA Clin. 2016;6:87-99.

42. Liang $\mathrm{D}$, Zeng $\mathrm{Q}$, Xu Z, et al. BAFF activates Erk $1 / 2$ promoting cell proliferation and survival by $\mathrm{Ca}^{2+}-\mathrm{CaMKII}$-dependent inhibition of PP2A in normal and neoplastic B-lymphoid cells. Biochem Pharmacol. 2014;87: 332-43.

43. Liu J, Sun Y. The role of PP2A-associated proteins and signal pathways in microcystin-LR toxicity. Toxicol Lett. 2015;236:1-7.

44. Gutierrez A, Pan L, Groen RW, et al. Phenothiazines induce PP2A-mediated apoptosis in T cell acute lymphoblastic leukemia. J Clin Invest. 2014;124:644-55.

\section{Submit your next manuscript to BioMed Central} and we will help you at every step:

- We accept pre-submission inquiries

- Our selector tool helps you to find the most relevant journal

- We provide round the clock customer support

- Convenient online submission

- Thorough peer review

- Inclusion in PubMed and all major indexing services

- Maximum visibility for your research

Submit your manuscript at www.biomedcentral.com/submit 